\title{
Theoretical Prediction of Intrinsic Self-Trapping of Electrons and Holes in Monoclinic $\mathrm{HfO}_{2}$
}

\author{
D. Muñoz Ramo, ${ }^{1, *}$ A. L. Shluger, ${ }^{1}$ J. L. Gavartin, ${ }^{2}$ and G. Bersuker ${ }^{3}$ \\ ${ }^{1}$ Department of Physics and Astronomy, University College London and London Centre for Nanotechnology, \\ Gower Street, London WC1E 6BT, United Kingdom \\ ${ }^{2}$ Accelrys, 334 Cambridge Science Park, Cambridge, CB4 OWN, United Kingdom \\ ${ }^{3}$ SEMATECH, 2706 Metropolis Dr., Austin, Texas 78741, USA
}

(Received 17 July 2007; published 12 October 2007)

\begin{abstract}
We predict, by means of $a b$ initio calculations, stable electron and hole polaron states in perfect monoclinic $\mathrm{HfO}_{2}$. Hole polarons are localized on oxygen atoms in the two oxygen sublattices. An electron polaron is localized on hafnium atoms. Small barriers for polaron hopping suggest relatively high mobility of trapped charges. The one-electron energy levels in the gap, optical transition energies and ESR $g$-tensor components are calculated.
\end{abstract}

PACS numbers: $61.72 . \mathrm{Bb}, 61.72 . \mathrm{Ji}, 71.15 . \mathrm{Mb}, 71.20 .-\mathrm{b}$

Control over carrier mobility in semiconductors and insulators under charge injection, photogeneration, or doping conditions is of enormous practical importance. In particular, the possibility of small electron and hole polaron formation in perfect deformable lattice (otherwise called self-trapping) has been considered in many studies (see Refs. [1-4] for recent reviews). However, proving polaron self-trapping remains extremely challenging. Essentially, the subject of debate is whether the carrier trapping takes place at preexisting precursor states (shallow donor, acceptor, or defect states, disorder fluctuations) or whether the injected carriers self-trap in a perfect lattice, where the potential well is only created by the carrierinduced lattice polarization.

Experimentally, distinguishing between trapping at defect sites and self-trapping in the perfect lattice is difficult due to crystal imperfection and generally small values of polaron self-trapping and migration energies. Reliable theoretical predictions, on the other hand, are rare, due to extreme sensitivity of the interplay between potential and kinetic energy of a polaron to a chosen Hamiltonian and boundary conditions (see, for example, Refs. [5,6]). The spectroscopic properties and diffusion barrier have been calculated for a number of systems, such as the selftrapped hole in alkali halides ( $V_{k}$ center) $[2,4]$, electron polaron in $\mathrm{TiO}_{2}$ [7], and for a number of predominantly hole small polarons trapped at impurities (see, for example, Ref. [8]) and in amorphous silica [9].

In this Letter we predict the self-trapping of both electrons and holes in the perfect monoclinic hafnium oxide $\left(m-\mathrm{HfO}_{2}\right)$ using static approach and density functional theory. $\mathrm{HfO}_{2}$ has been in the spotlight of both scientists and engineers over the last ten years as a potential substitute for $\mathrm{SiO}_{2}$ as a gate oxide in metal-oxidesemiconductor field-effect transistors (MOSFETs) [10]. The electron trapping in the dielectric layer in these devices may lead to degradation of their performance and reliability. The properties of the bulk $\mathrm{HfO}_{2}$ are very similar to those of $\mathrm{ZrO}_{2}$, which has much wider abundance and range of applications. The primitive unit cell of $m-\mathrm{HfO}_{2}$ (space group $P 2_{1} / c$ ) contains 12 atoms and two anion sublattices: in one oxygen ions are threefold coordinated (3C) and in the other-fourfold coordinated (4C).

Recent theoretical calculations predicted polaronlike electron trapping near neutral oxygen vacancies in $m-\mathrm{HfO}_{2}$ [11-13] and in the hypothetical amorphous $\mathrm{HfO}_{2}$ [14]. Here we investigate whether electrons and/or holes could be also trapped intrinsically by the perfect lattice. We address this question by calculating the polaron self-trapping energies, diffusion barriers, and spectroscopic properties in the framework of density functional theory (DFT). We have used the hybrid B3LYP functional, which includes nonlocal Fock exchange [15] and has been used successfully to model hole trapping in crystalline and amorphous $\mathrm{SiO}_{2}$ [9] and electron localization in an inorganic electride [16].

We use two complementary computational schemes implemented in periodic and embedded cluster methods. Periodic DFT calculations were performed with a parallel version of the CRYSTAL03 package [17], which uses local basis sets of Gaussian-type orbitals (GTOs) and the hybrid B3LYP functional. This method gave reliable predictions of defect properties in $m$ - $\mathrm{HfO}_{2}$ [11] and in other insulators $[16,18]$. We used an all electron basis set on oxygen ions and a relativistic effective core pseudopotential with a basis set for the outer electrons on Hf developed for this system in previous works [11]. The calculations were carried out in a 96-atom supercell with a MonkhorstPack mesh of $9 k$ points in the irreducible part of the Brillouin zone used for integration in the reciprocal space. A uniform background potential method was used in the calculations of charged supercells [17].

To facilitate the hole or electron localization, we created some small distortion around particular sites in the periodic cell and then allowed the whole system to relax. To study localization of electrons, we displaced two neighboring hafnium atoms by $0.2 \AA$ towards each other. In the case of holes, we elongated the Hf-O bonds of the correspond- 
ing oxygen atom by $0.1 \AA$, similar to the $V^{+}$oxygen vacancy. We have found a stable electron polaron state and two hole polaron states. The holes are localized on the 3C and $4 \mathrm{C}$ oxygen sites, respectively. In the case of the electron polaron, about $70 \%$ of the electron spin density is localized predominantly on three $\mathrm{Hf}$ ions sharing the $4 \mathrm{C}$ oxygen (see Fig. 1). It is distributed asymmetrically among these three ions, in a 40:20:10\% proportion. The rest of the spin density is delocalized over the more distant Hf ions. The lattice distortion is fairly small; the $4 \mathrm{C}$ oxygen ion shared by these three Hf ions is displaced by $0.2 \AA$ towards the fourth $\mathrm{Hf}$ ion.

The hole polaron predominantly localizes on a single oxygen site in both $3 \mathrm{C}$ and $4 \mathrm{C}$ oxygen sublattices. The hole localization on a $3 \mathrm{C}$ oxygen atom is accompanied by an outward displacement of the nearest neighbor (NN) Hf atoms by about $0.1 \AA$, and smaller displacements of the NN oxygen atoms (see Fig. 1). $74 \%$ of the spin density is on the oxygen atom, and three of the NN oxygen atoms trap a 9, a 7, and a $4 \%$ of density, respectively, (see Fig. 1). The remaining spin density is delocalized over the other oxygen atoms of the lattice. In the case of self-trapping on a $4 \mathrm{C}$ oxygen site, the lattice distorts in such a way that one of the O-Hf distances increases by $0.4 \AA$ and the other two Hf-O distances increase by about $0.2 \AA$. The NN oxygen atoms have smaller displacements, of the order of $0.1 \AA$. This atomic configuration is very similar to that obtained for the hole self-trapped on the $3 \mathrm{C}$ oxygen. $70 \%$ of the spin density is localized on the displaced oxygen atom, with $11 \%$ of the density on one of the NN O atoms (see Fig. 1).

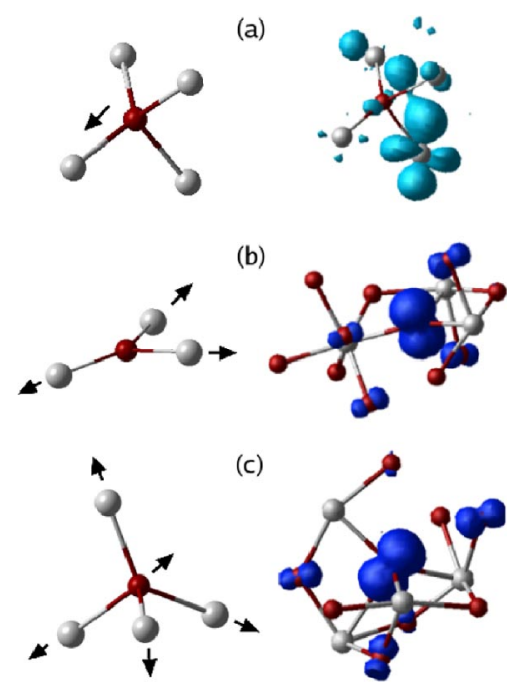

FIG. 1 (color online). Scheme of the lattice relaxation and spin localization in the three polaronic states. Left part shows the lattice relaxation and right part shows an isosurface (value $=$ 0.007 ) of the spin distribution in the area of the polaron. (a) corresponds to a self-trapped electron, (b) to a self-trapped hole in 3C oxygen, and (c) to a self-trapped hole in $4 \mathrm{C}$ oxygen. Bright balls represent $\mathrm{Hf}$ atoms and dark balls represent $\mathrm{O}$ atoms.
The self-trapping energies, $E_{\mathrm{ST}}$, are calculated as a difference between the total energies of the fully relaxed localized polaron state and the delocalized electron or hole state in the perfect undistorted lattice. They are summarized in Table I. The energy of the relaxed localized state is lower in all cases. In order to check that the localized states calculated in this work are not an artifact of the B3LYP functional we performed test calculations for $\mathrm{MgO}$, where no self-trapped holes have been found in the perfect lattice $[2,4]$. The geometry of a 64 -atom supercell with the same oxygen basis set was distorted towards the hypothetical one- or two- center hole configurations [19]. In both cases the lattice relaxed into the perfect geometry and the hole uniformly delocalized over the supercell. This suggests that the B3LYP functional is well balanced and does not artificially favor hole localization in similar oxides and the effect we observe in $\mathrm{HfO}_{2}$ may be genuine.

Figure 2(a) shows the density of states in the gap region for all polarons. The band gap of $6.1 \mathrm{eV}$ calculated for the perfect lattice is not affected by the polaron presence. The hole polaron, both in $3 \mathrm{C}$ and in $4 \mathrm{C}$ oxygen, creates an unoccupied localized state deep in the gap split by about $1 \mathrm{eV}$ from the valence band (VB) maximum. This state consist mainly from $p$ orbitals of the oxygen atom on which the polaron is localized. The electron polaron induces an occupied localized state split by $0.5 \mathrm{eV}$ from the conduction band (CB), and is mainly composed of a combination of $d$ orbitals of the three Hf atoms on which the electron is localized. The dispersion of polaron bands in both cases does not exceed $0.1 \mathrm{eV}$, in line with strong polaron localization.

The polaron mobility can be characterized by adiabatic barriers for hopping between different sites. To estimate these barriers we used a linear interpolation approach (see also Ref. [7]). Let $\mathbf{R}_{1}$ and $\mathbf{R}_{2}$ be the coordinates of all atoms in the initial and final polaron states, respectively. Then the coordinates of all atoms along the hopping path can be approximated as $\mathbf{R}=t \mathbf{R}_{1}+(1-t) \mathbf{R}_{2}$, where $t$ is varied in the range from 0 to 1 . Calculating energies for several configurations allows us to determine the highest energy along the adiabatic path and thus estimate a barrier. In Table I we present the barriers for polaron jumps be-

TABLE I. Self-trapping energies $\left(E_{\mathrm{ST}}\right)$, diffusion barriers for jumps to equivalent $\left(E_{d, e}\right)$ and nonequivalent $\left(E_{d, n}\right)$ sites, optical ionization $\left(E_{I}\right)$ and optical excitation $\left(E_{e}\right)$ energies, in $\mathrm{eV}$, and ESR $g$-tensor components for electron polaron and two different hole polaron states in $m$-HfO ${ }_{2}$. Negative oxygen vacancy calculated in the previous work [11] is included for comparison.

\begin{tabular}{lcccccccc}
\hline \hline State & $E_{\mathrm{ST}}$ & $E_{d, e}$ & $E_{d, n}$ & $E_{I}$ & $E_{e}$ & $g_{x x}$ & $g_{y y}$ & $g_{z z}$ \\
\hline Hole 3C & 0.57 & 0.08 & 0.56 & 1.21 & 1.51 & 2.014 & 2.025 & 2.040 \\
Hole 4C & 0.14 & 0.19 & 0.16 & 0.96 & 1.15 & 2.009 & 2.037 & 2.043 \\
Electron & 0.32 & 0.05 & - & 0.46 & 1.47 & 1.812 & 1.829 & 1.922 \\
$V^{-}$ & - & - & - & 1.24 & 0.78 & 1.811 & 1.829 & 1.941 \\
\hline \hline
\end{tabular}


(a)

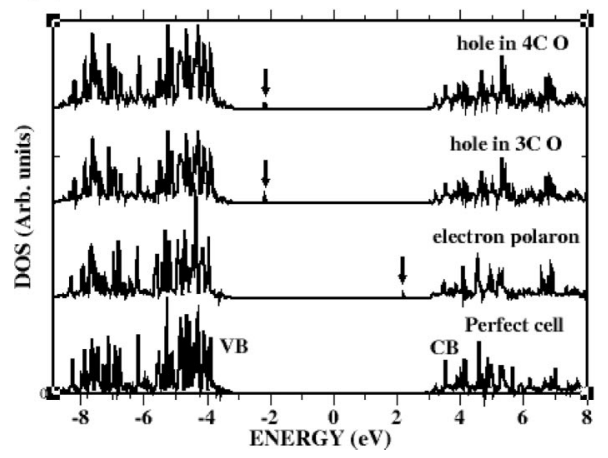

(b)
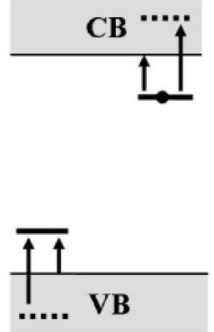

FIG. 2. (a) Density of states of $m-\mathrm{HfO}_{2}$ with the polaron states in the gap region marked by arrows. (b) Schematic of the optical transitions involving hole and electron polarons. Resonant states are indicated by dotted lines.

tween equivalent lattice sites, e.g., from $3 \mathrm{C}$ to $3 \mathrm{C}$ oxygen sites, and the barriers for jumps between nonequivalent, e.g., $3 \mathrm{C}$ and $4 \mathrm{C}$ oxygen sites. The barriers are quite low in almost all cases considered, with one notable exceptionthe barrier for the $3 \mathrm{C}-4 \mathrm{C}$ hole jump is much higher than that for the opposite process. Together with the larger selftrapping energy this indicates that a preferential pathway for hole diffusion in $m-\mathrm{HfO}_{2}$ structure is along oxygen $3 \mathrm{C}$ sites.

To further characterize the polaron states we have calculated optical ionization energies $\left(E_{I}\right.$, see Table I). For that purpose we calculate the difference in the total energies of the fully relaxed polaron state and that of the state in which the electron or hole are delocalized at the bottom of the $\mathrm{CB}$ (electron) or the top of the VB (hole), far from its original site, but the lattice atoms are still in the original polaron positions. We note that thermal ionization energies are well approximated by self-trapping energies, $E_{\mathrm{ST}}$. As expected [1], they are much smaller than $E_{I}$.

Optical absorption energies and ESR parameters are among the most important polaron fingerprints $[2,4]$. To calculate these properties we used an embedded cluster model implemented in the GUESS computer code described in Ref. [11]. A quantum (QM) cluster with the polaron at the center includes 13 hafnium ions and 26 oxygen ions. It is embedded in a spherically shaped nanocluster with monoclinic symmetry containing 2016 classical rigid ions and having a radius of approximately $25 \AA$. An interface between quantum and classical regions is described by substituting classical Hf atoms within a radius of $10 \AA$ from the polaron by large-core Hay and Wadt pseudopotentials [20]. The latter substitute all but four electrons of a hafnium atom. This prevents an artificial polarization of the electron density towards positive point ions outside the quantum cluster. The point ions outside the quantum cluster carry formal charges and contribute to the electrostatic potential within the quantum cluster (see Ref. [11] for more detail). We used the same GTO basis sets, pseudo- potentials, and B3LYP density functional as in the periodic calculations. The coordinates of the ions surrounding the relaxed polaron obtained in the CRYSTAL calculations were used also in embedded cluster calculations without further geometry optimization. The time-dependent (TD) DFT method was employed for calculating optical transition energies and $g$-tensor components were calculated using the technique implemented in the GAUSSIAN 03 package [21].

The electronic structure of $m-\mathrm{HfO}_{2}$ obtained in this model is in agreement with the periodic calculations. The band gap is by about $0.5 \mathrm{eV}$ larger than that in the CRYSTAL calculations and the splitting of the polaron states from the bands are also larger by about $0.5 \mathrm{eV}$ for the hole and $0.3 \mathrm{eV}$ for the electron polaron. These trends have also been observed in the previous embedded cluster study of the oxygen vacancy in $m$-HfO ${ }_{2}$ [11]. Using TDDFT we have calculated the relevant optical transitions: from the states in the VB to the localized unoccupied hole state in the gap, and from the electron polaron state in the gap to the unoccupied states in the CB [see Fig. 2(b)]. For each of these two types, 20 transitions have been calculated. The onset of hole transitions is at $0.4 \mathrm{eV}$ in the case of the $3 \mathrm{C}$ hole and at $0.3 \mathrm{eV}$ in the case of the $4 \mathrm{C}$ hole. The highest oscillator strength transitions are at $1.51 \mathrm{eV}(3 \mathrm{C}$ hole $)$ and $1.15 \mathrm{eV}$ ( $4 \mathrm{C}$ hole). These transitions take place from the oxygen ions surrounding that carrying the hole and forming a resonant state in the VB. The onset of electron polaron excitations is at $0.20 \mathrm{eV}$ and the maximum oscillator strength transition is at $1.47 \mathrm{eV}$. Similarly to the hole, this transition is to a resonant state composed of $d$ orbitals of $\mathrm{Hf}$ ions from the next coordination sphere.

The calculated $g$-tensor components for all polaron are shown in Table I. The value for the hole has little dependence on the site where it is self-trapped. It has orthorhombic symmetry, although the differences between components are small. The electron polaron shows a smaller value of the $g$ tensor and larger anisotropy in one of the components. In addition, the $g$-tensor components for this state are very similar to those calculated for the negatively charged oxygen vacancy [11], supporting the notion that the negatively charged vacancy is in fact an electron polaron trapped near a neutral vacancy.

In conclusion, we predict the existence of both hole and electron small polarons in $m-\mathrm{HfO}_{2}$. Holes can self-trap on a single oxygen atom in either of the two different sublattices of $m$ - $\mathrm{HfO}_{2}$, and electrons can self-trap on three $\mathrm{Hf}$ ions sharing an oxygen atom. This is a remarkable result, as the existence of both types of polarons is not common in insulators (see, for example, Ref. [22]). The low symmetry of $m-\mathrm{HfO}_{2}$, with two different oxygen sublattices stacked in alternate 2D slabs, combined with the high dielectric constant may facilitate the hole polaron self-trapping. The bottom of the $\mathrm{CB}$, composed mainly of narrow $d$ bands of Hf favors the electron localization, similar to other tran- 
sition metal oxides [23]. Experimental and theoretical works point out to the ability of the Zr-vacancy complex in yttrium-stabilized cubic $\mathrm{ZrO}_{2}$ to trap an electron and form the so-called $\mathrm{Zr}^{3+}$ center [24,25]. In this complex, the $\mathrm{Zr}$ atom lowers its coordination from eightfold to sevenfold or sixfold, which is similar to the Hf arrangement in the $m-\mathrm{HfO}_{2}$ lattice. Therefore the sevenfold coordination of $\mathrm{Hf}$ can be another factor in favor of the electron self-trapping.

Our results suggest that both hole and electron polarons in $m$ - $\mathrm{HfO}_{2}$ could be observed as stable immobile defects at low temperatures and should remain localized and exhibit hopping mobility at relatively high temperatures. Therefore, the hole and electron conductivity of pure $m$ - $\mathrm{HfO}_{2}$ samples should exhibit a characteristic temperature dependence [1]. It is interesting to note that the electronic conductivity of $\mathrm{ZrO}_{2}$ samples demonstrates activation energies in the range between 0.03 and $0.26 \mathrm{eV}$ [26,27]. These are similar to the barriers for polaron hopping obtained in our calculations of $m-\mathrm{HfO}_{2}$.

Apart from purely fundamental importance, these findings may have implications for our understanding of the performance of $\mathrm{HfO}_{2}, \mathrm{ZrO}_{2}$ and possibly other so-called high- $k$ oxides in microelectronic devices and in other applications. We note that an electron migration process exhibiting a very low activation energy of $0.01 \mathrm{eV}$ has recently been identified by simulating temperature dependent threshold voltage shift during constant voltage stress of the $\mathrm{HfO}_{2}$ gate dielectric MOSFETs using temperatureassisted charge migration model [28]. This low activation energy process might represent the transport of selftrapped electron polarons. Finally, we note that the existence of polaron bands should be taken into account in defining band offsets with respect to $\mathrm{Si}$ and other gate materials $[29,30]$. Self-trapping of both holes and electrons strongly suggests that excitons in this material would also self-trap. This may have important implications for its radiation stability [4].

This work was funded by EPSRC Grant No. GR/ S80080/01. The computer time on the HPCx facility was awarded to the Materials Chemistry consortium under EPSRC Grant No. GR/S13422/01 "Materials Chemistry using Teraflop Computing." We are grateful to A.M. Stoneham and V. V. Afanas'ev for useful discussions.

*Corresponding author. d.ramo@ucl.ac.uk

[1] I. G. Austin and N. F. Mott, Adv. Phys. 50, 757 (2001).

[2] R.T. Song and R.T. Williams, Self-Trapped Excitons (Springer-Verlag, Berlin Heidelberg New York, 1993).

[3] A. S. Alexandrov and N.F. Mott, Rep. Prog. Phys. 57, 1197 (1994).
[4] A. M. Stoneham and N. Itoh, Materials Modification by Electronic Excitation (Cambridge University Press, Cambridge, 2000).

[5] G. Pacchioni, F. Frigoli, D. Ricci, and J. A. Weil, Phys. Rev. B 63, 054102 (2000).

[6] J.L. Gavartin, P. V. Sushko, and A. L. Shluger, Phys. Rev. B 67, 035108 (2003).

[7] N. A. Deskins and M. Dupuis, Phys. Rev. B 75, 195212 (2007).

[8] O. F. Schirmer, J. Phys. Condens. Matter 18, R667 (2006).

[9] A. V. Kimmel, P. V. Sushko, and A. L. Shluger, J. NonCryst. Solids 353, 599 (2007).

[10] High-k Gate Dielectrics: why do we need them?, edited by M. Houssa (IOP Publishing, Bristol and Philadelphia, 2004).

[11] D. Muñoz Ramo, J.L. Gavartin, A. L. Shluger, and G. Bersuker, Phys. Rev. B 75, 205336 (2007).

[12] K. Xiong, J. Robertson, M. C. Gibson, and S. G. Clark, Appl. Phys. Lett. 87, 183505 (2005).

[13] P. Broqvist and A. Pasquarello, Appl. Phys. Lett. 89, 262904 (2006).

[14] J.L. Gavartin, D. Muñoz Ramo, A.L. Shluger, and G. Bersuker, ECS Trans. 3, 277 (2006).

[15] A. D. Becke, J. Chem. Phys. 98, 1372 (1993).

[16] P. V. Sushko, A. L. Shluger, M. Hirano, and H. Hosono, J. Am. Chem. Soc. 129, 942 (2007).

[17] V. Saunders, R. Dovesi, C. Roetti, R. Orlando, C. M. Zicovich-Wilson, N. M. Harrison, K. Doll, B. Civalleri, B. Bush, and P.L.M. D'Arco, CRYSTAL 2003 User's Manual (University of Torino, Torino, 2003).

[18] F. Cora, M. Alfredsson, G. Mallia, D. S. Middlemiss, W. C. Mackrodt, R. Dovesi, and R. Orlando, Principles and Applications of Density Functional Theory in Inorganic Chemistry II, Structure and Bonding (Berlin) Vol. 113 (Springer, Berlin, 2004), p. 171.

[19] J. L. Gavartin and A. L. Shluger, Phys. Rev. B 64, 245111 (2001).

[20] P. J. Hay and W. R. Wadt, J. Chem. Phys. 82, 270 (1985).

[21] M. J. Frisch et al., GAUSSIAN 03 Revision C02 (Gaussian Inc., Wallingford, CT, 2003).

[22] S. V. Nistor, E. Goovaerts, and D. Schoemaker, Phys. Rev. B 57, 1 (1998).

[23] N. S. P. Bhuvanesh and J. Gopalakrishnan, J. Mater. Chem. 7, 2297 (1997).

[24] F. Pietrucci, M. Bernasconi, C. Di Valentin, F. Mauri, and C. J. Pickard, Phys. Rev. B 73, 134112 (2006).

[25] V. M. Orera, R. I. Merino, Y. Chen, R. Cases, and P. J. Alonso, Phys. Rev. B 42, 9782 (1990).

[26] M. Levy, J. Fouletier, and M. Kleitz, J. Electrochem. Soc. 135, 1584 (1988).

[27] D. Eder and R. Kramer, Phys. Chem. Chem. Phys. 8, 4476 (2006).

[28] G. Bersuker, J. H. Sim, S. Park, C. D. Young, S. Nadkami, R. Choi, and B. H. Lee, IEEE Trans Dev. Mat. Reliability 7, 138 (2007).

[29] E. M. Conwell, Synth. Met. 83, 101 (1996).

[30] I. H. Campbell, T. W. Hagler, D. L. Smith, and J.P. Ferraris, Phys. Rev. Lett. 76, 1900 (1996). 